ARTícUlO

\title{
Uso de organismos intermareales del litoral rocoso como bio-indicadores de deformación continental cosísmica en el centro sur de Chile
}

\author{
Use of rocky shore intertidal organisms as bioindicators of coseismic continental deformation in south \\ central Chile
}

\section{Pamela López $^{1 *}$ y Eduardo Jaramillo ${ }^{1}$}

\begin{abstract}
${ }^{1}$ Instituto de Ciencias de la Tierra, Facultad de Ciencias, Universidad Austral de Chile, Edificio Emilio Pugín, Avenida Eduardo Morales Miranda, Campus Isla Teja, Valdivia, Chile. Autor corresponsal: *pamelalopez.san@gmail.com

Abstract.- On February 27, 2010, the south central coast of Chile (ca., 34-39 ${ }^{\circ} \mathrm{S}$ ) was affected by an earthquake magnitude $\mathrm{Mw}=8,8$, with the epicenter located offshore Cobquecura $\left(c a ., 36.29^{\circ} \mathrm{S}\right)$. This earthquake originated continental uplift on that coastal areas closer to the trench between the Nazca and South American tectonic plates (i.e., Peninsula de Arauco and Isla Santa María) and subsidence along coastal areas located further north of that (the coast of the Maule Region), as well as in continental zones ordered in a west - east axis. In this study we tested the hypothesis that independent of the taxonomic group, the rocky shore sessile benthic organisms can be used as indicators of continental uplift. The methodology involved measurements of heights upper of distributional bands above the low tide level at the coast affected by the earthquake. The results show that the calcareous crustose algae Lithothamnium sp., the mytilid bivalve Perumytilus purpuratus and the macroalgae Mazzaella laminarioides and Lessonia spicata, are benthonic useful organisms to describe continental uplift, due to the fact that their vertical distributions after the earthquake follow the coastal deformation observed in the field. This allow to suggest that in studies aimed to evaluate this tectonic processes, more than a single species must be used in order to gain a full view of that along gradients of continental deformation. This due to the fact that a significant number of the benthic species of the rocky shore do not have continuous distribution; thus, to use a single species may well result in conclusions with low level of generalizations.
\end{abstract}

Key words: Continental uplift, benthic organisms, subsidence

Resumen.- El 27 de febrero de 2010, la zona centro-sur de Chile (ca., 34-39 $\mathrm{S}$ ) fue afectada por un terremoto de magnitud $\mathrm{Mw}=8,8$, localizándose el epicentro en Cobquecura $\left(c a ., 36^{\circ} \mathrm{S}\right)$, causando un levantamiento continental en las costas más cercanas a la fosa (i.e., Península de Arauco e Isla Santa María) y subsidencia en áreas costeras localizadas al norte de esas costas (Región del Maule). En este estudio se evaluó la hipótesis de que independientemente del grupo taxonómico, los organismos bentónicos intermareales adheridos al sustrato pueden ser utilizados como indicadores de levantamiento cosísmico continental. La metodología consistió en medir la altura del límite superior de las bandas de distribución de cada especie sobre el nivel de marea baja, a lo largo de la costa afectada por el terremoto. El alga calcárea Lithothamnium sp., el mitílido Perumytilus purpuratus y las macroalgas Mazzaella laminarioides y Lessonia spicata, son organismos bentónicos apropiados para describir el levantamiento continental cosísmico. Se sugiere que en estudios tendientes a evaluar este tipo de procesos tectónicos, se utilice más de una especie para tener una visión completa de tal proceso a lo largo de gradientes de deformación continental. Esto ya que, parte importante de las especies bentónicas de la costa rocosa no tienen distribución continúa, por lo que el uso de una sola especie puede resultar en conclusiones con bajo nivel de generalización.

Palabras clave: Levantamiento continental, organismos bentónicos, subsidencia

\section{INTRODUCCIÓN}

Chile es uno de los países con mayor actividad sísmica en el mundo (Farías et al. 2010, Madariaga et al. 2010), debido a que convergen dos placas tectónicas, la de Nazca y la Sudamericana. Esta convergencia entre estas placas es de aproximadamente $6,8 \mathrm{~cm}$ año-1 (Ruegg et al. 2009), lo cual origina grandes sismos de magnitudes entre 8 y 9 Mw cada aproximadamente 100-150 años (Lomnitz 2004) y > $9 \mathrm{Mw}$ cada aproximadamente 300 años (Cisternas et al. 2005). 
La madrugada del 27 de febrero del 2010 (27F de aquí en adelante), la costa del centro sur de Chile (ca., 34$39^{\circ} \mathrm{S}$ ) fue afectada por un terremoto de magnitud $\mathrm{Mw}=8,8$ (Farías et al. 2010). Hasta ese momento, este evento fue el quinto más grande registrado de modo instrumental en el mundo y ocurrió en la laguna sísmica más extensa que existía en Chile hasta el verano del 2010 y que estaba comprendida entre Pichilemu por el norte (ca., $34^{\circ} \mathrm{S}$ ) y Concepción por el sur (ca., 37º $)$. En esa zona, no habían ocurrido grandes sismos ( $\geq 8,0 \mathrm{Mw}$ ) desde el 20 de febrero de 1835 (Farías et al. 2010).

El epicentro del 27F se ubicó bajo el mar y frente a la localidad costera de Cobquecura (ca., 36º S), localizándose el hipocentro aproximadamente a $30 \mathrm{~km}$ de profundidad (Vargas et al. 2011). El área de fractura de la placa estuvo comprendida entre Valparaíso (ca., 33으) por el norte e Isla Mocha (ca., $39^{\circ} \mathrm{S}$ ) por el sur y abarcó aproximadamente $610 \mathrm{~km}$, con un desplazamiento cercano a los $10 \mathrm{~m}$ en el contacto inter placa (Quezada et al. 2010).

El sismo del 27F ocasionó un levantamiento continental cosísmico (especialmente conspicuo en la costa de la península de Arauco e Isla Santa María) y subsidencia en la zona costera ubicada al norte de esas costas (i.e., Región del Maule) y en áreas continentales ordenadas en un eje oeste-este; los mayores alzamientos alcanzaron valores promedio cercanos $250 \mathrm{~cm}$, lo que afectó la infraestructura costera y remodeló el paisaje litoral (Castilla et al. 2010, Farías et al. 2010, Quezada et al. 2010, Jaramillo et al. 2012, Rodil et al. 2015, 2016).

Estudios realizados en diferentes litorales rocosos, muestran que la deformación continental cosísmica producto de un terremoto de subducción afectó a la flora y fauna litoral (e.g., Plafker \& Savage 1970, Ortlieb et al. 1996, Wong 2009, Morton et al. 2011). En aquellas áreas emergidas, ocurre mortandad masiva de macroalgas e invertebrados marinos, algunos de los cuales han sido utilizados como marcadores de levantamiento continental, incluyendo cirripedios (Plafker \& Savage 1970), bivalvos (Barrow 1836, Castilla et al. 2010) y algas calcáreas incrustantes (Corallinaceae) (Castilla et al. 2010, Farías et al. 2010, Quezada et al. 2010, Jaramillo et al. 2012). Sin embargo, no se ha analizado en general, el uso de varios de esos organismos de modo coincidental, aun cuando el estudio de Ortega et al. (2014) analizó el efecto del levantamiento continental del $27 \mathrm{~F}$ sobre un conjunto de especies de invertebrados que ocurre en grampones del alga parda Lessonia spicata.
En este estudio, se evaluó la hipótesis que, independientemente del grupo taxonómico, los organismos bentónicos intermareales adheridos al sustrato rocoso, sirven como bio-indicadores de levantamiento cosismico continental en la costa del centro-sur de Chile. Para ello, se estudió la variabilidad en los límites superiores de la distribución vertical de algas e invertebrados bentónicos de amplia distribución en las costas del Maule y Biobío, afectadas por el 27F. Con el objetivo de evaluar la magnitud de esa deformación, se realizaron mediciones similares en la costa de Valdivia y Osorno, donde aparentemente no fue observada alguna deformación continental durante este terremoto.

\section{MATERIALES Y MÉTODOS}

\section{ÁREA DE ESTUDIO}

En la Figura 1 y Tabla S1, se muestran la ubicación geográfica de los sitios de estudio en la Región del Maule, Biobío, Los Ríos y Los Lagos, Chile. En la Tabla S2, se muestran los códigos diseñados para la identificación de los mismos así como otros términos abreviados que se mencionan en el texto.

\section{MEDICIONES EN TERRENO}

Los datos utilizados en este estudio, están basados en estimaciones de la altura del límite superior de la distribución vertical de organismos bentónicos en la zona intermareal rocosa con respecto al nivel relativo del mar post- terremoto 27F. Las distribuciones de los organismos estudiados incluyen los siguientes taxa: i) un alga calcárea incrustante perteneciente a la familia Corallinaceae (Rhodophyta, Cryptonemiales) (Lithothamnium sp. y comúnmente distribuida en los niveles inferiores del litoral rocoso de la costa chilena. Cuando viva, esta alga tiene un característico color rosado que pasa a blanco debido a una exposición extrema a la luz solar y altas temperaturas (Ortlieb et al. 1996, Ramírez-Herrera \& Orozco 2002). Si bien en este estudio no se determinó a que género realmente pertenece esta alga, se le ha denominado Lithothamnium sp. (Heydrich, 1897) debido a que esta denominación ha sido ampliamente utilizada en estudios recientes similares a este (Ortlieb et al. 1996, Farías et al. 2010, Quezada et al. 2010, Vargas et al. 2011); ii) el bivalvo mitílido Perumytilus purpuratus (Lamarck, 1819), especie dominante de amplia distribución geográfica en los niveles 


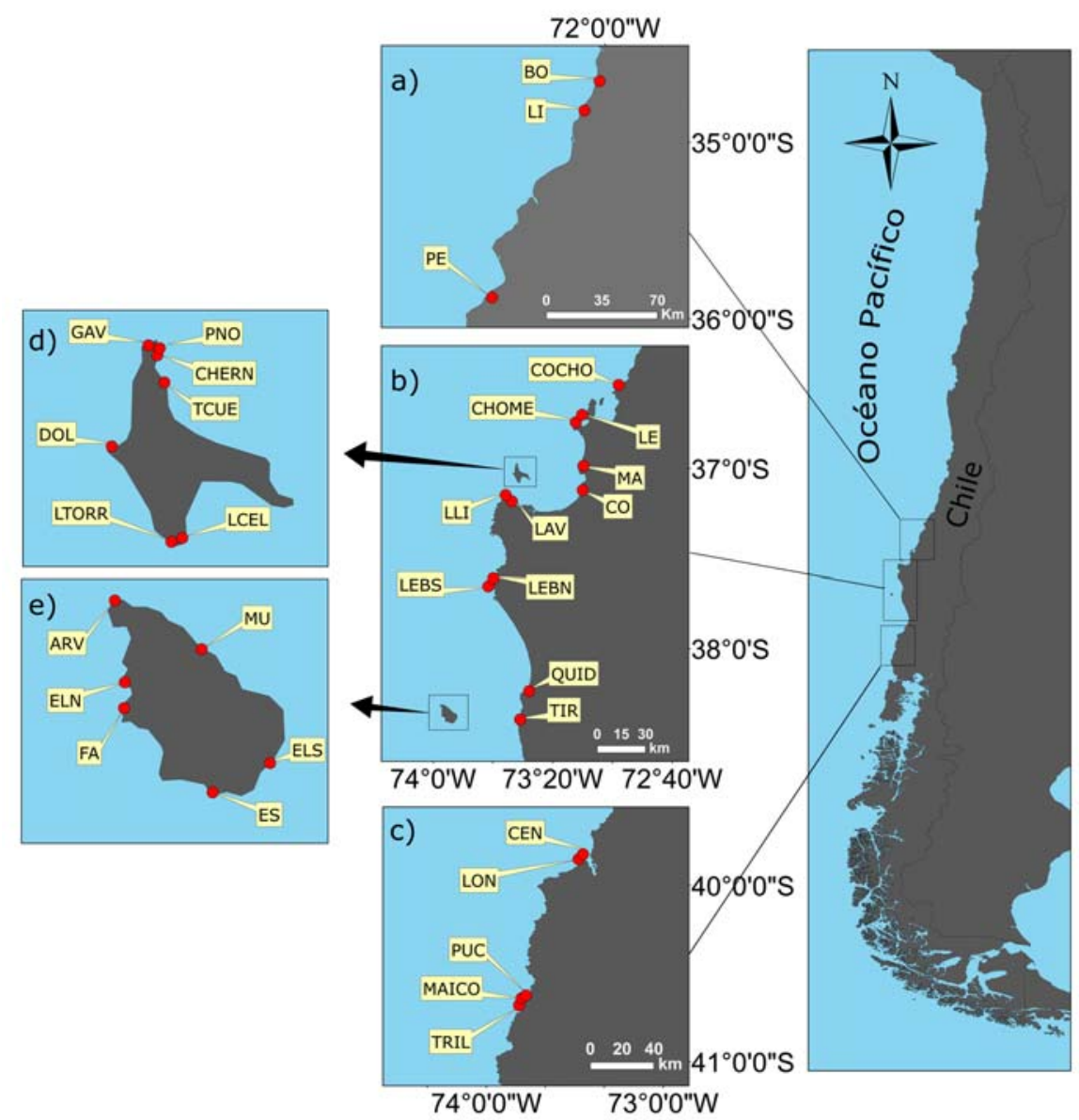

Figura 1. Localización de los sitios de muestreos en la costa: a) Región del Maule, b) Región del Biobío, c) Regiones de Los Ríos y de Los Lagos, d) Isla Santa María (ISM) y e) Isla Mocha (IM). En la Tabla S1 se indican las latitudes y longitudes de esos sitios y en la Tabla S2 el significado de los códigos indicados en esta figura / Location of sampling sites on the coast: a) Maule Region, b) Biobío Region, c) Los Ríos and Los Lagos Regions, d) Isla Santa María and e) Isla Mocha. Table S1 shows latitudes and longitudes of those sites and Table S2 shows the meaning of codes indicated in this figure

medios del intermareal rocoso de la costa chilena (Castilla 1981); iii) la macroalga roja Mazzaella laminarioides, (Bory de Saint-Vincent, 1828) Fredericq, especie que en general ocurre en límites intermareales ubicados bajo el cinturón de Perumytilus purpuratus, y iv) la macroalga parda Lessonia spicata (Suhr) Santelices comb. nov., especie típica del intermareal rocoso inferior de la costa de Chile. De estos taxa, las especies más representadas (i.e., mayor número de sitios donde ocurra una especie) fueron Lithothamnium sp. y Perumytilus purpuratus (Fig. 2).
Las mediciones se establecieron a través de estimaciones de la altura del límite superior de la banda de distribución de cada especie en relación al nivel relativo del mar representado por el nivel de la marea baja al momento de realizar las mediciones. Los muestreos se efectuaron entre abril y mayo del 2010 en la costa continental de las regiones del Maule y el Biobío, en noviembre 2010 en la Isla Santa María y Región de Los Lagos, en diciembre 2010 en Isla Mocha, y diciembre 2011 en la Región de Los Ríos. Los muestreos se efectuaron en períodos de mareas de sicigia. 

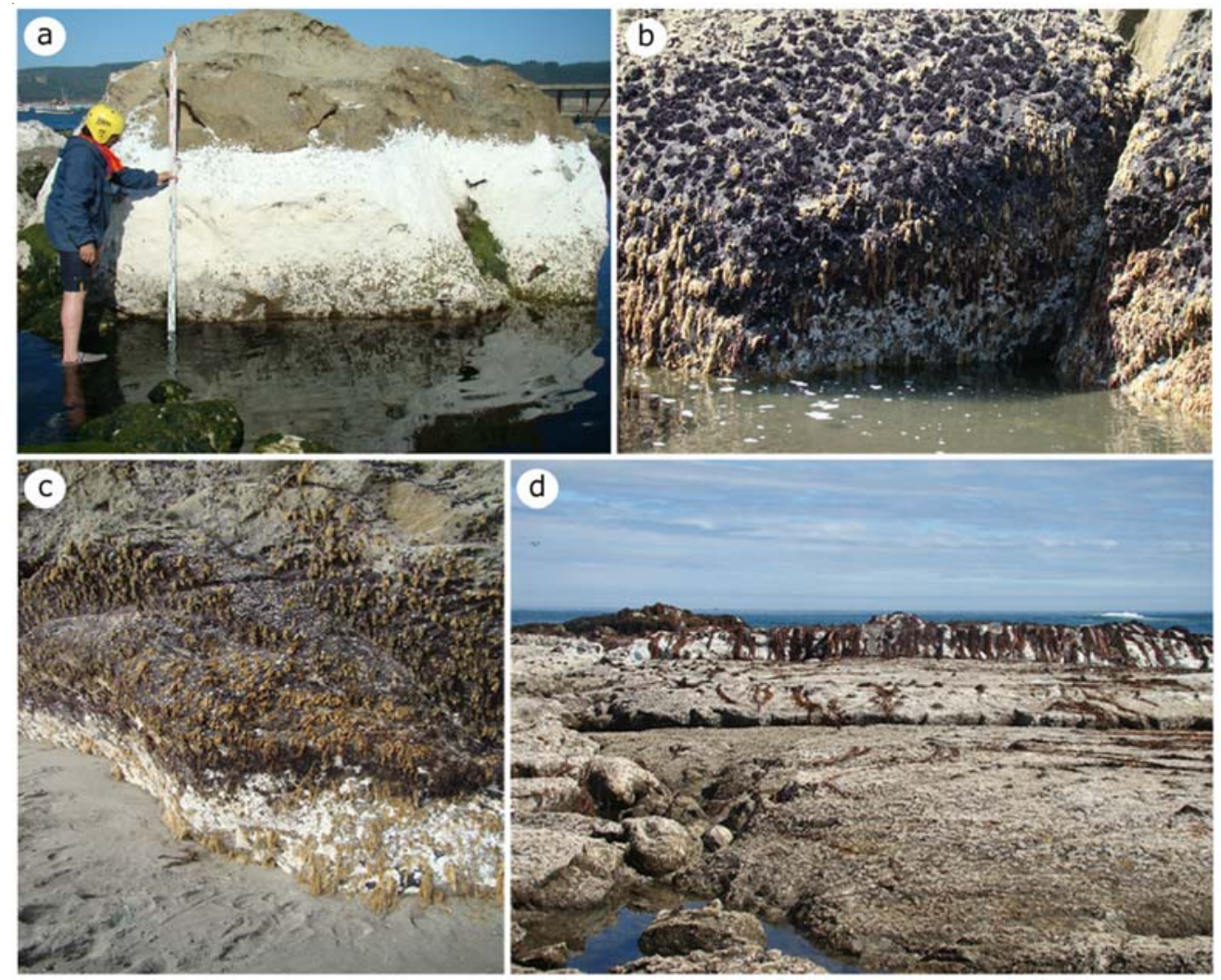

Figura 2. Efecto del levantamiento continental cosísmico de la costa rocosa durante el 27F. (a) roca blanqueada por la presencia de algas calcáreas incrustantes muertas (Lithothamnium sp.) en la costa de Lebu;(b) bivalvos (Perumytilus purpuratus) muertos en la Isla Santa María; (c) algas rojas muertas (Mazzaella laminarioides) en la costa de Lebu;(d) algas pardas muertas (Lessonia spicata) en Lebu / Effect of the coesismic continental uplift of the rocky shore during the 27F. (a) rocks bleached for the presence of dead calcareous crustose algae (Lithothamnium sp.) on the coast of Lebu; (b) dead bivalves (Perumytilus purpuratus) on Isla Santa María; (c) dead red algae (Mazzaella laminarioides) on the coast of Lebu; (d) dead brown algae (Lessonia spicata) on Lebu

\section{ANÁLISIS DE LOS DATOS}

Los datos de terreno se analizaron luego de corregir las mediciones por el efecto de marea de acuerdo a las alturas de estas. Para ello, se trabajó con diferentes paquetes computacionales, tales como MATLAB $\AA$ (The MathWorks, Inc.) y XTide (FlaterCo) ${ }^{1}$ a fin de permitir estandarizar y realizar una comparación de las alturas verticales de las especies con respecto al nivel medio del mar, a lo largo de la costa afectada por el terremoto. Los datos corregidos de las alturas de los límites superiores de las diferentes especies, se agruparon como sigue: i) costa del Maule, ii) costa del Biobío al norte de la Península de Arauco, iii) costa de la Península de Arauco e Isla Santa María, iv) costa de la Isla Mocha, y v) costa de Valdivia y Osorno. Los análisis estadísticos consistieron en comparar los promedios de las alturas de las especies en estudio de cada uno de esos grupos mediante análisis de varianza (ANDEVA); los datos no transformados fueron examinados para evaluar normalidad y homocedasticidad con los tests de Shapiro-Wilk y Levene, respectivamente. Luego, se aplicó el Test de Tukey para evaluar cuáles promedios diferían significativamente entre sí.

\section{Resultados}

La variabilidad espacial de los promedios de los límites superiores $(\mathrm{cm})$ de la banda vertical del alga calcárea incrustante Lithothamnium sp. se muestran en la Tabla 1 y Figura 3; se incluyen los límites superiores del alga blanqueada o muerta y del alga rosada o viva.

\footnotetext{
${ }^{1}<$ https://flaterco.com/xtide.html>
} 
Tabla 1. Variabilidad espacial en los promedios de los límites superiores $(\mathrm{cm})$ de la banda de Lithothamnium sp. en el área de estudio. $L s b=$ límite superior del alga blanqueada o muerta; $L s r=$ límite superior del alga rosada o viva. Los valores en paréntesis corresponden a desviación estándar. La ausencia de datos indica que no se detectó la presencia de especímenes de Lithothamnium sp., blanqueados o muertos / Spatial variability in the averages of the upper limits $(\mathrm{cm})$ of the band of Lithothamnium sp. in the study area. Lsb= upper limit of bleached or dead algae; Lsr= upper limit of pink or living algae. The values in parentheses correspond to standard deviation. The absence of data indicates that the presence of white or dead Lithothamnium sp., was not detected

\begin{tabular}{|c|c|c|c|}
\hline Región y áreas & Sitios & Lsb & Lsr \\
\hline \multirow[t]{3}{*}{ MAULE } & Boyeruca & - & $25,44(27,94)$ \\
\hline & Lipimávida & - & $75,50(-)$ \\
\hline & Pelluhue & $60,6(-)$ & \\
\hline \multirow{4}{*}{$\begin{array}{l}\text { BIOBÍO - al norte de la Península } \\
\text { de Arauco }\end{array}$} & Cocholhue & $74,78(15,70)$ & - \\
\hline & Chome & $125,68(82,38)$ & - \\
\hline & Maule & - & $59,7(85,32)$ \\
\hline & Colcura & - & $29,5(24,89)$ \\
\hline \multirow{6}{*}{$\begin{array}{l}\text { BIOBÍO - Península de Arauco y } \\
\text { cercanías (Quidico y Tirúa) }\end{array}$} & Lavapié & $180,17(61,38)$ & - \\
\hline & Llico & $217,56(32,05)$ & - \\
\hline & Lebu Norte & $215,76(55,36)$ & - \\
\hline & Lebu Sur & $214,67(26,76)$ & - \\
\hline & Quidico & $101,13(46,74)$ & - \\
\hline & Tirúa & $148,11(60,29)$ & - \\
\hline \multirow[t]{7}{*}{ BIOBIOO - Isla Santa María } & Punta Norte & $244,48(55,17)$ & - \\
\hline & Gaviotas & $262,68(44,09)$ & - \\
\hline & Caleta Hernández & $241,4(70,74)$ & - \\
\hline & Tres Cuevas & $170,67(52,77)$ & - \\
\hline & Dolores & $264,37(80,87)$ & - \\
\hline & Las Torres & $329,37(74,07)$ & - \\
\hline & La Celina & $234,7(51,60)$ & - \\
\hline \multirow[t]{3}{*}{ BIOBÍO - Isla Mocha } & El Natre & $103,86(31,67)$ & - \\
\hline & El Santiago & $18,83(22,53)$ & - \\
\hline & El Saco & $46,93(40,24)$ & - \\
\hline \multirow[t]{2}{*}{ LOS RIOOS - Valdivia } & Centinilla & - & $-0,56(21,75)$ \\
\hline & Loncoyén & - & $-7,89(23,64)$ \\
\hline \multirow[t]{3}{*}{ LOS LAGOS - Osorno } & Pucatrihue & & $85,14(41,31)$ \\
\hline & Maicolpué & - & $8,03(30,11)$ \\
\hline & Tril Tril & - & $30,38(32,91)$ \\
\hline
\end{tabular}

Lithothamnium sp.

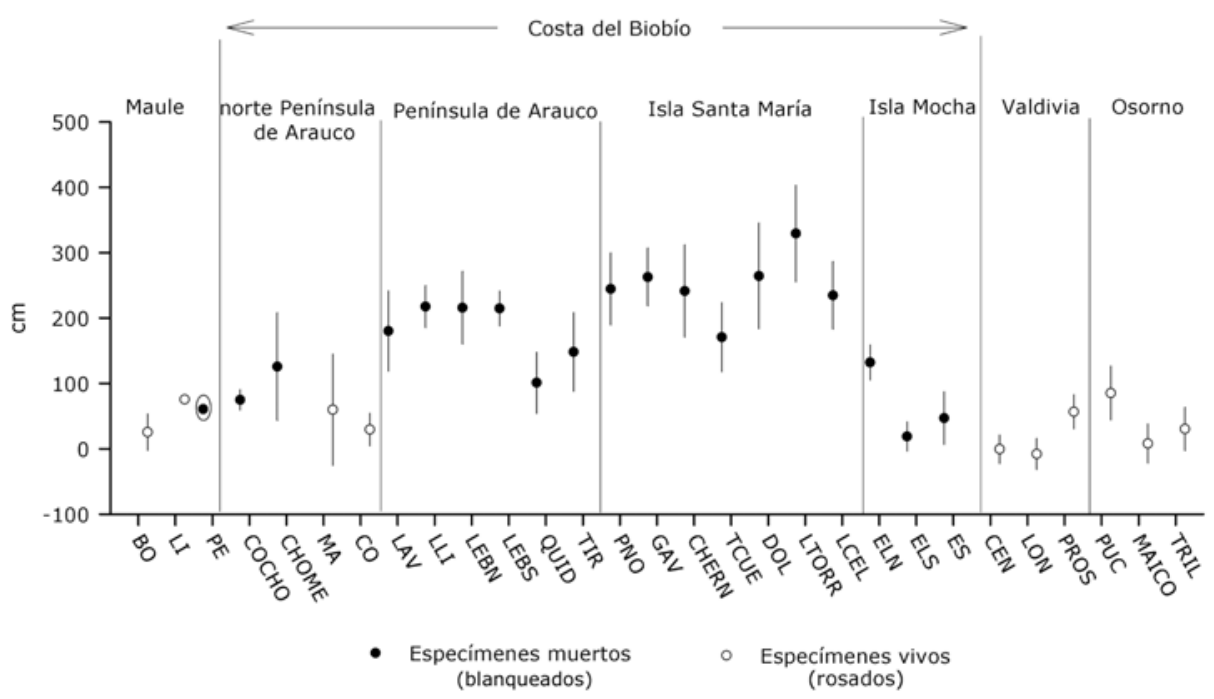

Figura 3. Variabilidad espacial de los límites superiores de la banda del alga calcárea incrustante Lithothamnium sp. en los sitios muestreados (cf. Tabla S2 para el significado de los códigos de cada sitio). Los valores son promedios \pm 1 desviación estándar. La elipse en color rojo indica la presencia de especímenes muertos en Pelluhue, situación probablemente no relacionada a deformación continental cosísmica (ver texto) / Spatial variability of the upper limits of the crustose calcareous algae Lithothamnium sp. on the sampling sites ( $c f$. Table S2 for meanings of site codes). The values are means \pm 1 standard deviation. The red ellipse indicates the presence of dead specimens at Pelluhue, a fact probably not related to coseismic land level changes (see text) 
Los resultados de los análisis ANDEVA obtenidos para evaluar eventuales diferencias entre áreas costeras, muestran que la banda de Lithothamnium sp. ocurrió a niveles significativamente más altos $(P<0,05)$ en los sitios muestreados en la Península de Arauco e Isla Santa María (especímenes muertos) en comparación con aquellos de la costa del Maule, costa al norte de la Península de Arauco, Isla Mocha, Valdivia y Osorno (Tabla 2).

La variabilidad espacial en los promedios de los límites superiores (cm) de la franja del bivalvo mitílido Perumytilus purpuratus se muestran en la Tabla 3 y Figura 4. Los resultados de ANDEVA, muestran que esos límites ocurrieron a alturas significativamente más altas $(P<0,05)$ en los sitios estudiados en la Península de Arauco e Isla Santa María (especímenes muertos; Tabla 4, cf. Fig. 4). Es más, no se registraron diferencias significativas $(P>0,05)$ al comparar con los sitios de la costa del Maule, costa al norte de la península de Arauco, Isla Mocha, Valdivia y Osorno (especímenes vivos). No se detectaron diferencias significativas $(P>0,05)$ al comparar estos últimos sitios entre sí (Tabla 4).
Tabla 2. Resultados de los análisis ANDEVA, realizados para evaluar eventuales diferencias en los límites superiores de la banda de especímenes blanqueados o muertos y rosados o vivos del alga calcárea incrustante Lithothamnium sp. Se presentan los valores de $P$ y q resultantes de los análisis de ANDEVA. Los valores en negrilla son significativos a un nivel de probabilidad de 0,05 / Results of ANOVA performed to evaluate eventual differences in the upper limits of the band of bleached or dead and pink or alive specimens of the crustose calcareous algae Lithothamnium sp. The values of $P$ and $q$ resulting from ANOVA are displayed. Values in bold are significant at a 0.05 probability level

\begin{tabular}{|c|c|c|c|}
\hline & Sitios & $P$ & $q$ \\
\hline \multirow[t]{5}{*}{ Costa de Maule (vivos) vs } & $\begin{array}{l}\text { Costa al norte de la Peninsula de } \\
\text { Arauco (vivos) }\end{array}$ & 0,947 & 1,425 \\
\hline & $\begin{array}{l}\text { Costa al norte de la Península de } \\
\text { Arauco (muertos) }\end{array}$ & 1,000 & 0,168 \\
\hline & $\begin{array}{l}\text { Costa Península de Arauco e Isla } \\
\text { Santa Maria (muertos) }\end{array}$ & 0,003 & 6,291 \\
\hline & Costa Isla Mocha (muertos) & 1,000 & 0,191 \\
\hline & Costa de Valdivia y Osorno (vivos) & 0,998 & 0,767 \\
\hline \multirow[t]{3}{*}{$\begin{array}{l}\text { Costa al norte de la Península } \\
\text { de Arauco (muertos) vs }\end{array}$} & $\begin{array}{l}\text { Costa Península Arauco e Isla Santa } \\
\text { Maria (muertos) }\end{array}$ & 0,002 & 6,512 \\
\hline & Costa Isla Mocha (muertos) & 1,000 & 0,375 \\
\hline & Costa de Valdivia y Osorno (vivos) & 0,576 & 2,512 \\
\hline Costa Península Arauco e Isla & Costa Isla Mocha (muertos) & $<0,001$ & 7,189 \\
\hline Santa María (muertos) vs & Costa de Valdivia y Osorno (vivos) & $<0,001$ & 10,950 \\
\hline Costa Isla Mocha (muertos) vs & Costa de Valdivia y Osorno (vivos) & 0,983 & 1,131 \\
\hline
\end{tabular}

Perumytilus purpuratus

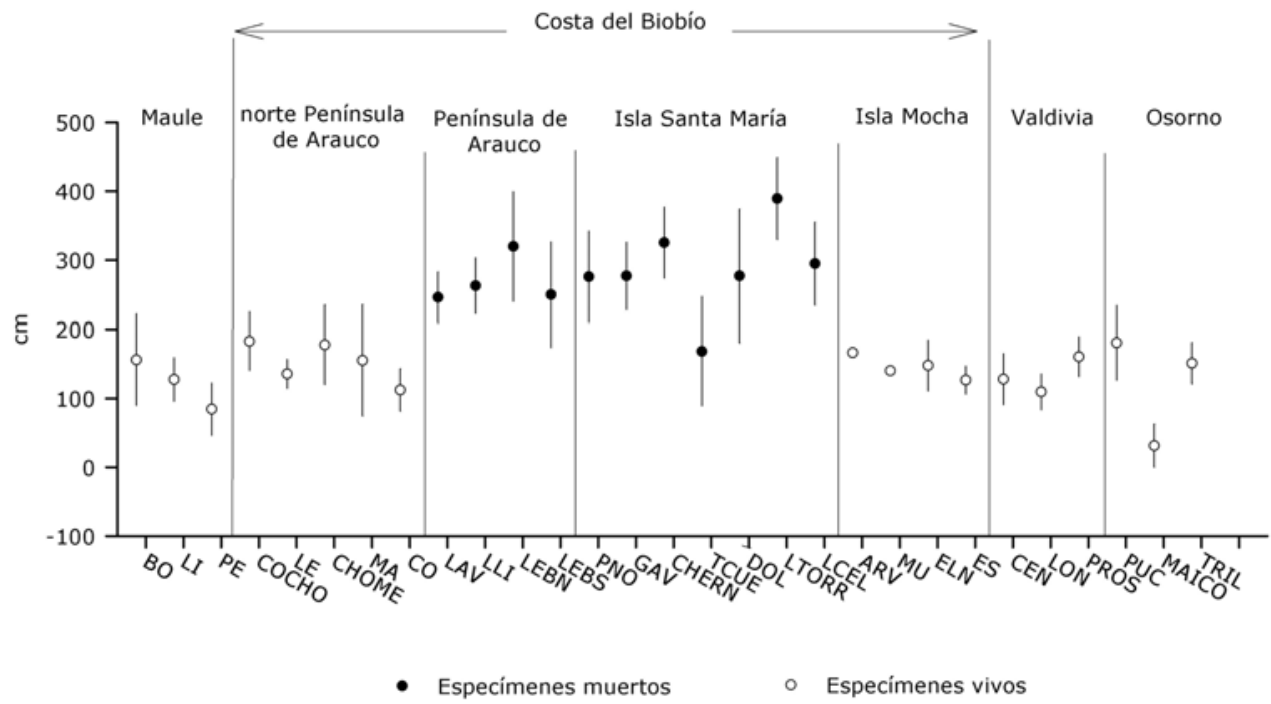

Figura 4. Variabilidad espacial de los límites superiores de la banda del bivalvo Perumytilus purpuratus en los sitios muestreados (cf. Tabla S2 para el significado de los códigos de cada sitio). Los valores son promedios $\pm \mathbf{1}$ desviación estándar / Spatial variability of the upper limits of the bivalve Perumytilus purpuratus on the sampling sites ( $c f$. Table S2 for meanings of site codes). The values are means \pm 1 standard deviation 
Tabla 3. Variabilidad espacial en los promedios de los límites superiores $(\mathrm{cm})$ de la banda de Perumytilus purpuratus. Ls= límite superior. Los valores en paréntesis corresponden a desviación estándar / Spatial variability in the average of the upper limits $(\mathrm{cm})$ of the band of Perumytilus purpuratus. Ls= upper limit. The values in parentheses are standard deviation

\begin{tabular}{llr}
\hline \multicolumn{1}{c}{ Región y áreas } & \multicolumn{1}{c}{ Sitios } & \multicolumn{1}{c}{ Ls } \\
\hline MAULE (vivos) & Boyeruca & $156,04(65,95)$ \\
& Lipimávida & $127,52(31,70)$ \\
& Pelluhue & $84,46(38,01)$ \\
BIOBíO - al norte de la Península & Cocholhue & $182,77(42,21)$ \\
de Arauco (vivos) & Lenga & $135,7(21,00)$ \\
& Chome & $177,68(57,39)$ \\
& Maule & $155,15(80,67)$ \\
& Colcura & $112,2(30,68)$ \\
BIOBíO - Peninsula de Arauco y & Lavapié & $246,07(36,51)$ \\
cercanías (Quidico y Tirúa) & Llico & $262,9(40,41)$ \\
(muertos) & Lebu Norte & $319,76(79,56)$ \\
& Lebu Sur & $250(76,36)$ \\
BIOBíO - Isla Santa María & Punta Norte & $275,8(66,03)$ \\
(muertos) & Gaviotas & $277,01(48,72)$ \\
& Caleta Hernández & $325,33(51,43)$ \\
& Tres Cuevas & $168,23(78,91)$ \\
& Dolores & $277,04(96,80)$ \\
& Las Torres & $389,37(59,44)$ \\
& La Celina & $294,84(60,20)$ \\
BIOBíO - Isla Mocha (vivos) & Arveja & $166,3(-)$ \\
& Muelle & $140,23(5,17)$ \\
& El Natre & $147,86(36,78)$ \\
& El Saco & $126,7(20,14)$ \\
& Centinilla & $128,04(36,89)$ \\
LOS RíOS - Valdivia (vivos) & Loncoyén & $109,61(25,88)$ \\
& Pucatrihue & $180,46(53,76)$ \\
LOS LAGOS - Osorno (vivos) & Maicolpué & $31,35(31,35)$ \\
& Tril Tril & $151,08(29,77)$ \\
\hline & &
\end{tabular}

Tabla 4. Resultados de los análisis ANDEVA, realizados para evaluar eventuales diferencias en los límites superiores de la banda del bivalvo mitílido Perumytilus purpuratus. Se presentan los valores de $P$ y $q$ resultantes del análisis de ANDEVA. Los valores en negrilla son significativos a un nivel de probabilidad de 0,05 / Results of ANOVA performed to evaluate eventual differences in the upper limits of the band of the bivalve Perumytilus purpuratus. The values of $P$ and $q$ resulting from ANOVA are displayed. Values in bold are significant at a 0.05 probability level

\begin{tabular}{llrr}
\hline & Sitios & $P$ & \multicolumn{1}{c}{$\mathrm{q}$} \\
\hline Costa de Maule (vivos) vs. & Costa al norte de la Península de & 0,899 & 1,254 \\
& $\begin{array}{l}\text { Arauco (vivos) } \\
\text { Costa Peninsula de Arauco e Isla }\end{array}$ & $<\mathbf{0 , 0 0 1}$ & $\mathbf{7 , 3 9 6}$ \\
& $\begin{array}{l}\text { Santa María (muertos) } \\
\text { Costa Isla Mocha (vivos) }\end{array}$ & 0,967 & 0,903 \\
& Costa de Valdivia y Osorno (vivos) & 1,000 & 0,179 \\
& Costa Península de Arauco e Isla & $<\mathbf{0 , 0 0 1}$ & $\mathbf{7 , 2 3 3}$ \\
Costa al norte de la Península & Santa Maria (muertos) & 0,999 & 0,338 \\
de Arauco (vivos) vs. & Costa Isla Mocha (vivos) & 0,886 & 1,304 \\
& Costa de Valdivia y Osorno (vivos) & $<\mathbf{0 0 1}$ & $\mathbf{7 , 0 7 0}$ \\
Costa Peninsula Arauco e Isla & Costa Isla Mocha (vivos) & $<\mathbf{0 7 0}$ \\
Santa María (muertos) vs & Costa de Valdivia y Osorno (vivos) & $<\mathbf{0 , 0 0 1}$ & $\mathbf{9 , 2 4 3}$ \\
Costa Isla Mocha (vivos) vs & Costa de Valdivia y Osorno (vivos) & 0,971 & 0,872 \\
\hline
\end{tabular}

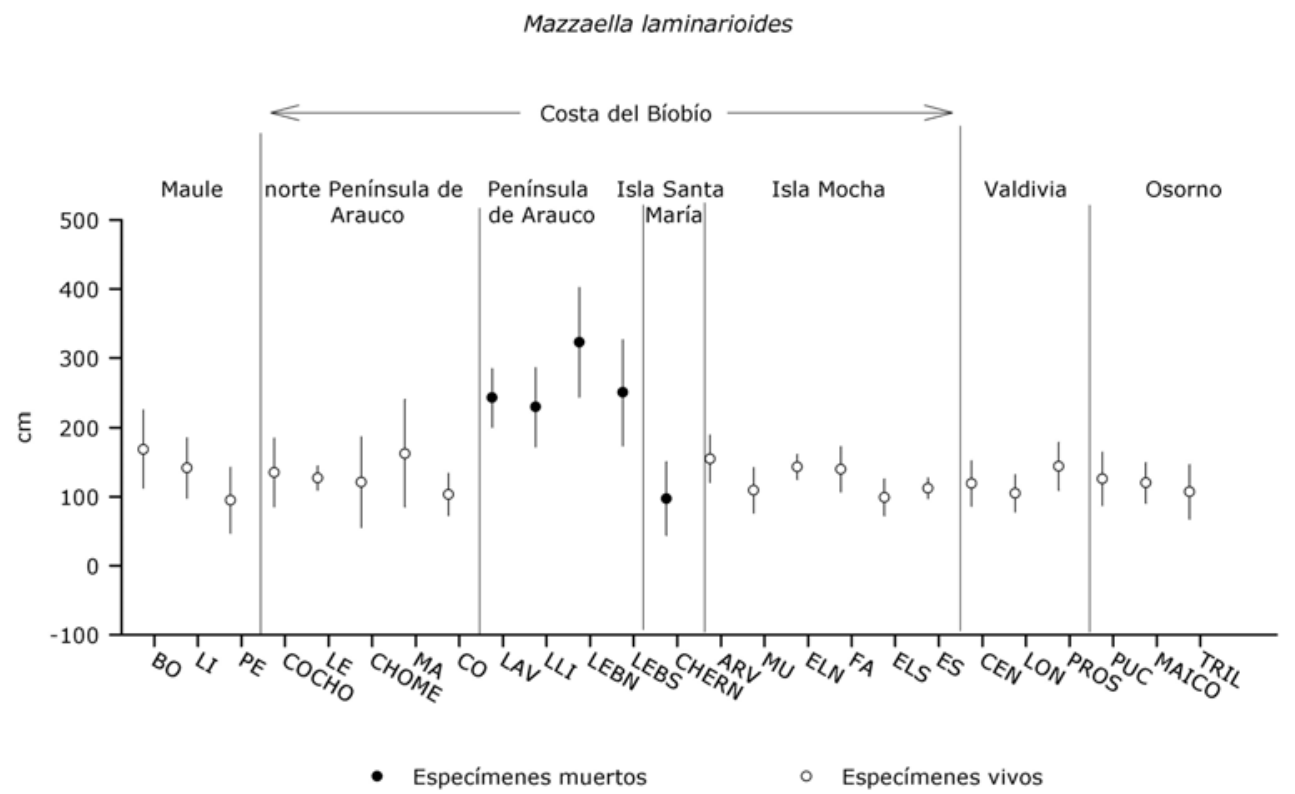

Figura 5. Variabilidad espacial de los límites superiores del alga roja Mazzaella laminarioides en los sitios muestreados (cf. Tabla S2 para el significado de los códigos de cada sitio) / Spatial variability of the upper limits of the red algae Mazzaella laminarioides on the sampling sites (cf. Table S2 for meanings of site codes). The values are means \pm 1 standard deviation 
Tabla 5. Variabilidad espacial en los promedios del límite superior (cm) de la banda de especímenes del alga roja Mazzaella laminarioides. Ls= límite superior. Los valores en paréntesis corresponden a desviación estándar / Spatial variability in the average of the upper limits $(\mathrm{cm})$ of the band of specimens of the red algae Mazzaella laminarioides. Ls= upper limit. The values in parentheses are standard deviation

\begin{tabular}{llr}
\hline \multicolumn{1}{c}{ Región y áreas } & \multicolumn{1}{c}{ Sitios } & \multicolumn{1}{c}{ Ls } \\
\hline MAULE (vivos) & Boyeruca & $168,44(56,04)$ \\
& Lipimávida & $141,52(43,72)$ \\
& Pelluhue & $94,8(47,72)$ \\
BIOBÍO - al norte de & Cocholhue & $135,02(49,65)$ \\
Península de la Arauco & Lenga & $126,95(17,46)$ \\
(vivos) & Chome & $120,98(65,64)$ \\
& Maule & $162,3(77,10)$ \\
& Colcura & $103,2(30,52)$ \\
BIOBíO - Península de & Lavapié & $242,28(41,85)$ \\
Arauco y cercanías (Quídico & Llico & $228,9(56,91)$ \\
y Tirúa) (muertos) & Lebu Norte & $322,6(79,40)$ \\
& Lebu Sur & $250(76,36)$ \\
BIOBíO - Isla Santa María & Caleta Hernández & $97,10(53,03)$ \\
(muertos) & & \\
BIOBíO - Isla Mocha & Arveja & $154,81(34,38)$ \\
(vivos) & Muelle & $109,2(32,92)$ \\
& El Natre & $143,18(18,10)$ \\
& Faro & $139,74(32,88)$ \\
& El Santiago & $98,95(26,46)$ \\
& El Saco & $112,11(15,09)$ \\
LOS RÍOS - Valdivia & Centinilla & $119,04(32,86)$ \\
(vivos) & Loncoyén & $104,81(27,11)$ \\
LOS LAGOS - Osorno & Pucatrihue & $125,76(38,61)$ \\
(vivos) & Maicolpué & $120,03(29,22)$ \\
& Tril Tril & $106,98(39,51)$ \\
\hline & & \\
& &
\end{tabular}

La variabilidad espacial en los promedios de los límites superiores $(\mathrm{cm})$ de la distribución vertical del alga roja Mazzaella laminarioides se muestran en la Tabla 5 y Figura 5. Los resultados de ANDEVA, revelan que los límites superiores de esta especie en la Península de Arauco e Isla Santa María fueron significativamente más altos $(P<0,05)$ que en la costa al norte de la Península de Arauco, Isla Mocha, Valdivia y Osorno (especímenes vivos; Tabla 6).

La variabilidad espacial en los límites superiores (cm) de la distribución vertical del alga parda Lessonia spicata en el área de estudio se muestran en la Tabla 7 y Figura 6. Los resultados de ANDEVA muestran que la banda de esta especie se observó a mayor altura $(P<0,05)$ en los sitios muestreados en la Península de Arauco e Isla Santa María vs. aquellos sitios ubicados al norte de la Península de Arauco, Valdivia y Osorno (especímenes vivos). No se detectaron diferencias significativas $(P>0,05)$ al comparar las alturas promedio de la banda con las otras zonas entre sí ( $c f$. Tabla 8 y Fig. 6).

Tabla 6. Resultados de los análisis ANDEVA, realizados para evaluar eventuales diferencias en los límites superiores de la banda de especímenes del alga roja Mazzaella laminarioides. Se muestran los valores de $\boldsymbol{P}$ y q resultantes del análisis de ANDEVA. Los valores en negrilla son significativos a un nivel de probabilidad de 0,05 / Results of ANOVA performed to evaluate eventual differences in the upper limits of the band of specimens of the red algae Mazzaella laminarioides. The values of $P$ and $q$ resulting from ANOVA are displayed. Values in bold are significant at a probability level of 0.05

\begin{tabular}{llcc}
\hline & Sitios & $P$ & $\mathrm{q}$ \\
\hline Costa de Maule (vivos) vs. & $\begin{array}{l}\text { Costa al norte de la Península de } \\
\text { Arauco (vivos) }\end{array}$ & 1,000 & 0,242 \\
& $\begin{array}{l}\text { Costa Península de Arauco e Isla } \\
\text { Santa María (muertos) }\end{array}$ & $\mathbf{0 , 0 4 4}$ & $\mathbf{4 , 3 2 0}$ \\
& Costa Isla Mocha (vivos) & 0,998 & 0,411 \\
& Costa Valdivia y Osorno (vivos) & 0,986 & 0,710 \\
Costa al norte de la Península & Costa Península de Arauco e Isla & $\mathbf{0 , 0 1 1}$ & $\mathbf{5 , 2 6 8}$ \\
Arauco (vivos) vs. & Santa María (muertos) & 1,000 & 0,188 \\
& Costa Isla Mocha (vivos) & 0,995 & 0,537 \\
Costa Península de Arauco e & Costa de Valdivia y Osorno (vivos) & $\mathbf{0 , 0 0 5}$ & $\mathbf{5 , 6 9 0}$ \\
Isla Santa María (muertos) vs. & Costa de Valdivia y Osorno (vivos) & $\mathbf{0 , 0 0 3}$ & $\mathbf{6 , 0 3 9}$ \\
Costa Isla Mocha (vivos) vs. & Costa de Valdivia y Osorno (vivos) & 0,999 & 0,366 \\
\hline
\end{tabular}




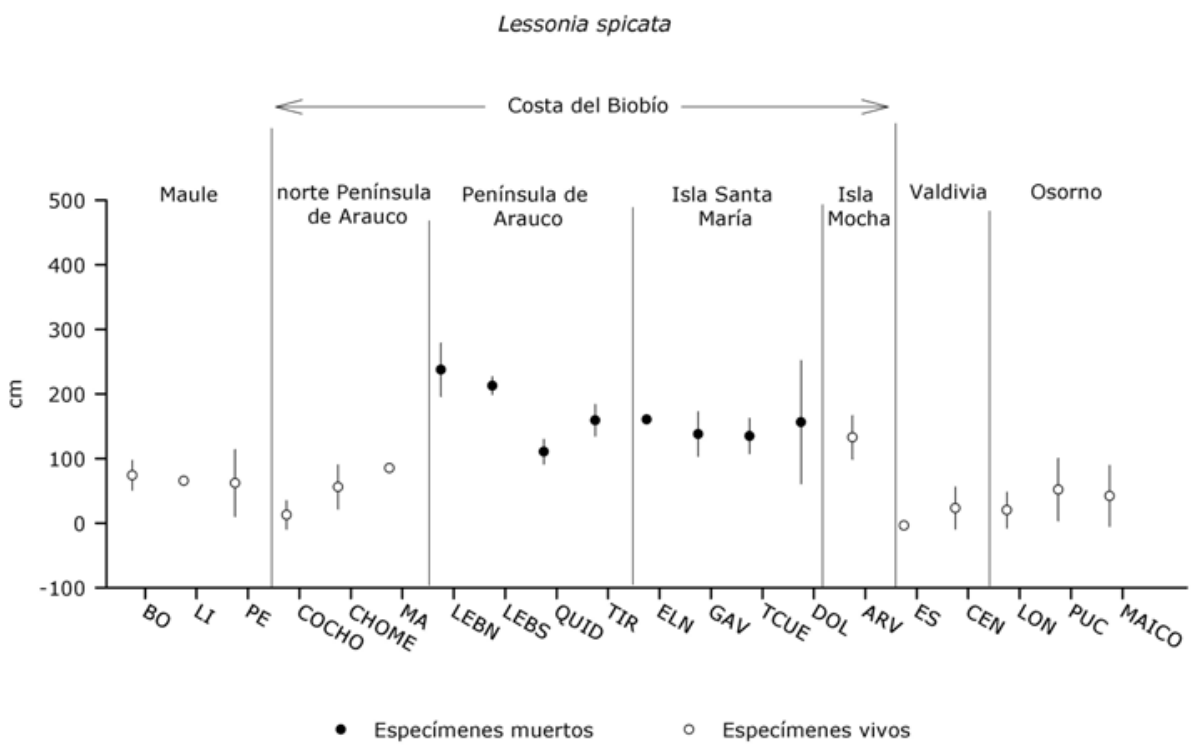

Figura 6. Variabilidad espacial de los límites superiores del alga café Lessonia spicata en los sitios muestreados (cf. Tabla S2 para el significado de los códigos de cada sitio). Los valores son promedios \pm 1 desviación estándar / Spatial variability of the upper limits of the brown algae Lessonia spicata on the sampling sites (cf. Table S2 for meanings of site codes). The values are means \pm 1 standard deviation

Tabla 7. Variabilidad espacial en los promedios de los límites superiores $(\mathrm{cm})$ de la banda de especímenes del alga parda Lessonia spicata. Ls= límite superior. Los valores en paréntesis corresponden a desviación estándar / Spatial variability in the average of the upper limits $(\mathrm{cm})$ of the band of specimens of the brown seaweed Lessonia spicata. Ls= upper limit. The values in parentheses are standard deviation

\begin{tabular}{llr}
\hline \multicolumn{1}{c}{ Región y áreas } & \multicolumn{1}{c}{ Sitios } & \multicolumn{1}{c}{ Ls } \\
\hline MAULE (vivos) & Boyeruca & $74,04(23,04)$ \\
& Lipimávida & $65,52(7,50)$ \\
& Pelluhue & $61,96(51,63)$ \\
BIOBÍO - al norte de la Península & Cocholhue & $12,7(22,00)$ \\
de Arauco (vivos) & Chome & $55,93(34,20)$ \\
& Maule & $85,10(-)$ \\
BIOBíO - Península de Arauco y & Lebu Norte & $237,56(41,16)$ \\
cercanías (Quidico y Tirúa) & Lebu Sur & $212,78(13,84)$ \\
(muertos) & Quidico & $110,52(19,20)$ \\
& Tirúa & $159,25(24,36)$ \\
BIOBíO - Isla Santa María & Punta Norte & $160,5(-)$ \\
(muertos) & Gaviotas & $137,83(34,58)$ \\
& Tres Cuevas & $134,86(27,37)$ \\
& Dolores & $156,34(95,37)$ \\
BIOBÍO - Isla Mocha (vivos) & El Saco & $-3,70(-)$ \\
LOS RíOS - Valdivia (vivos) & Centinilla & $23,24(32,62)$ \\
& Loncoyén & $20,11(27,71)$ \\
LOS LAGOS - Osorno (vivos) & Pucatrihue & $51,76(48,35)$ \\
& Maicolpué & $41,94(47,22)$ \\
& Tril Tril & $55,38(27,02)$ \\
\hline
\end{tabular}

Tabla 8. Resultados de los análisis ANDEVA, realizados para evaluar eventuales diferencias entre los límites superiores de la banda de especímenes del alga parda Lessonia spicata. Se presentan los valores de $\boldsymbol{P}$ y $q$ resultantes del análisis de ANDEVA. Los valores en negrilla son significativos a un nivel de probabilidad de 0,05 / Results of ANOVA performed to evaluate eventual differences in the upper limit of the band of specimens of the brown alga Lessonia spicata. The values of $P$ and q resulting from ANOVA are displayed. Values in bold are significant at a probability level of 0.05

\begin{tabular}{|c|c|c|c|}
\hline & Sitios & $P$ & $q$ \\
\hline \multirow[t]{3}{*}{ Costa de Maule (vivos) vs. } & $\begin{array}{l}\text { Costa al norte de la Península de } \\
\text { Arauco (vivos) }\end{array}$ & 0,974 & 0,843 \\
\hline & $\begin{array}{l}\text { Costa Península de Arauco e Isla } \\
\text { Santa Maria (muertos) }\end{array}$ & 0,004 & 6,164 \\
\hline & Costa de Valdivia y Osorno (vivos) & 0,751 & 1,698 \\
\hline \multirow[t]{2}{*}{$\begin{array}{l}\text { Costa al norte de la Península } \\
\text { de Arauco (vivos) vs. }\end{array}$} & $\begin{array}{l}\text { Costa Península de Arauco e Isla } \\
\text { Santa María (muertos) }\end{array}$ & 0,001 & 7,181 \\
\hline & Costa de Valdivia y Osorno (vivos) & 0,982 & 0,755 \\
\hline $\begin{array}{l}\text { Costa Peninsula de Arauco e } \\
\text { Isla Santa Maria (muertos) vs. }\end{array}$ & Costa de Valdivia y Osorno (vivos) & $<0,001$ & 9,496 \\
\hline
\end{tabular}




\section{Discusión}

Este estudio estuvo enfocado en evaluar algunos organismos bentónicos pertenecientes a diferentes taxa como bio-indicadores de levantamiento cosísmico continental en la costa rocosa intermareal del centro sur de Chile. El uso de algunos de estos organismos ha sido común desde las observaciones pioneras de Robert FitzRoy, comandante del 'HMS Beagle', cuando en conjunto con Charles Darwin describieran los efectos del terremoto de 1835 en las costas de la Isla Santa María y del Biobío en Chile (Barrow 1836, Darwin 1851, Lee 2010). Los resultados de este estudio, muestran que todas las especies analizadas resultaron ser buenos indicadores para estimar la deformación continental, ya que en la zona donde ocurrió levantamiento cosísmico, estas especies tuvieron modificaciones en los límites superiores de su distribución vertical, a la vez que en las costas no afectadas por la deformación continental (i.e., Valdivia y Osorno) estos taxa no mostraron variaciones en sus zonaciones verticales.

Uno de los organismos analizados en este estudio fue Lithothamnium sp., alga calcárea perteneciente a la familia Corallinaceae (Meneses 1993) y abundante en la zona intermareal baja y submareal de la costa rocosa chilena. La distribución vertical de esta especie es controlada primariamente por el oleaje (Farías et al. 2010); sus paredes celulares poseen carbonato de calcio lo que le permite sobrevivir al pastoreo de invertebrados ramoneadores (Ortlieb et al. 1996). Las plantas de Lithothamnium sp. tienen un característico color rosado, el que cambia a blanco cuando quedan expuestas al aire y la desecación. De ahí que la altura vertical de esta alga en el litoral rocoso ha sido utilizada como expresión de levantamiento continental cosísmico (Ortlieb et al. 1996, Farías et al. 2010, Castilla et al. 2010).

Los resultados de este estudio muestran que los valores promedios de los límites superiores de la distribución vertical de la banda blanqueada de Lithothamnium sp. (i.e., especímenes muertos) fueron significativamente más altos $(P<0,05)$ en los sitios estudiados en la Península de Arauco e Isla Santa María versus aquellos ubicados más al norte o al sur de esa área y donde hubo menor deformación continental o donde se observó subsidencia como en la costa de Maule (Jaramillo et al. 2012). En el sitio de Pelluhue (Región del Maule), se observó una banda de Lithothamnium sp. blanqueada, aun cuando este sitio se ubica en la zona de subsidencia continental por efecto del terremoto (Fig. 2). Esta situación podría estar asociada a la ocurrencia durante el período del oleaje (mar en calma) con mareas más bajas, con una constante exposición a altas temperatura y radiación solar, lo que puede haber provocado el blanqueamiento de Lithothamnium sp. en esta zona ( $c f$. Meneses 1993).

Otra de las especies analizadas fue el bivalvo mitílido Perumytilus purpuratus, organismo que ocupa gran parte del gradiente vertical en la zona intermareal rocosa del litoral chileno (Alvarado \& Castilla 1996, Broitman et al. 2001). Este bivalvo fue recientemente utilizado para evaluar el levantamiento cosísmico del terremoto $27 \mathrm{~F}$ (Melnick et al. 2012).

Las estimaciones entregadas por los datos de distribución vertical de esta especie en la costa afectada por el terremoto son en general coincidentes con los datos de deformación continental entregados por los GPS (Melnick et al. 2012).

Las observaciones realizadas en este estudio sobre la distribución vertical de los organismos muertos de Perumytilus purpuratus, muestran un patrón similar al observado en Lithothamnium sp., es decir, los valores promedios de los límites superiores de este bivalvo fueron significativamente más altos $(P<0,05)$ en los sitios localizados en la Península de Arauco e Isla Santa María, comparados con los demás sitios.

En general, no hay muchos estudios que estimen si macroalgas no incrustantes reflejan la variabilidad en altura que ocurre luego de un terremoto de subducción. El alga utilizada en este estudio fue Mazzaella laminarioides, macroalga roja correspondiente a la familia Gigartinaceae y que se distribuye a lo largo de la costa, extensamente en el intermareal medio y bajo (Santelices 1991). Su tamaño poblacional es afectado por procesos tales como los vinculados al evento ENOS (El Niño Oscilación del Sur), altas temperaturas y episodios como levantamientos continentales durante la ocurrencia de terremotos de subducción (Montecinos et al. 2012) siendo algunos de estos las causas de mortandades masivas. Los resultados para $M$. laminarioides muestran, que los valores promedios de los límites superiores fueron significativamente más altos $(P<0,05)$ en los sitios estudiados en la Península de Arauco comparados con el resto de los sitios.

El alga parda Lessonia spicata, antes conocida como Lessonia nigrescens, fue utilizada como bio-indicador de levantamiento continental cosísmico producto del terremoto del 3 de marzo de 1985 en la costa de Chile central (Castilla \& Oliva 1990). En este estudio, la banda 
vertical de distribución de esa macroalga se observó a alturas significativamente más altas $(P<0,05)$ en los sitios muestreados en la Península de Arauco e Isla Santa María, coincidiendo con la visible mortalidad detectada en terreno.

Los resultados anteriores permiten aceptar la hipótesis planteada en este estudio, ya que Lithothamnium sp., Perumytilus purpuratus y Lessonia spicata mostraron valores más altos de distribución vertical en la Península de Arauco e Isla Santa María, sitios con mayor levantamiento continental (Farías et al. 2010, Quezada et al. 2010). Conclusiones similares pueden derivarse para Mazzaella laminarioides, aun cuando especímenes muertos sólo se encontraron en el sitio Caleta Hernández en la Isla Santa María. Los datos anteriormente expuestos, coinciden con el área donde se registraron los mayores alzamientos continentales cosísmicos, con una elevación promedio de $250 \mathrm{~cm}$; es decir, la altura de los organismos marinos muertos en las rocas evidencia la magnitud del alzamiento continental en la Península de Arauco e Isla Santa María. En la Isla Mocha, el levantamiento fue de menor magnitud a pesar de la cercanía de la misma a la fosa que ocurre entre las placas de Nazca y Sudamericana (Quezada et al. 2010); la evidencia de esto proviene de las menores alturas a las que se observaron especímenes muertos de por ejemplo, Perumytilus purpuratus en la misma. Por último, no se observó evidencia de alzamientos cosísmicos en los sitios estudiados en las Regiones de Maule, Los Ríos (Valdivia) y Los Lagos (Osorno), concluyendo que los valores más altos en los límites superiores se observaron en la Península de Arauco e Isla Santa María, resultado del levantamiento continental cosísmico producto del 27F. El alga calcárea crustosa Lithothamnium sp. Resultó ser un buen indicador de levantamiento cosísmico, puesto que, el típico color rosado de la misma cambia a blanco producto de la emersión y exposición al aire. Sin embargo, esta misma alga se observó en la costa de la región de Coquimbo luego del sismo de Illapel del 16 de septiembre del 2015, específicamente en la localidad de Tongoy, donde no se detectó deformación continental aparente.

Los resultados de este trabajo pueden ser útiles para evaluar el efecto del eventual levantamiento continental cosísmico y recuperación de la costa rocosa luego de un terremoto de subducción en cualquier lugar de la costa chilena donde ocurran las especies aquí estudiadas. Sin embargo, se debe tener presente que muchas especies de invertebrados bentónicos del litoral rocoso muestran una notoria distribución en parches (Cerda \& Castilla 2001), resultado de heterogeneidad del sustrato, dinámica del asentamiento y reclutamiento (Camus \& Lagos 1996, Lagos et al. 2007). Se hace relevante entonces la necesidad de realizar observaciones y mediciones en conjuntos de especies y no taxa aislados a fin de obtener conclusiones con generalizaciones apropiadas.

\section{Agradecimientos}

A las personas que participaron en las mediciones en terreno: Mario Manzano, Cristian Duarte, Cesar Campos, Mauricio L' Huissier, Gonzalo Morales y Ronald Sánchez. Este estudio fue financiado por el proyecto FONDECYT $\mathrm{N}^{\circ} 1121043$ 'Coastal Legacies of the Maule earthquake in Chile: Ecological research needs and opportunities in the wake of the large natural disturbances', cuyo investigador responsable fue Eduardo Jaramillo.

\section{LITERATURA CITADA}

Alvarado JL \& JC Castilla. 1996. Tridimensional matrices of Perumytilus purpuratus on intertidal platforms with varying wave forces in central Chile. Marine Ecology Progress Series 133: $135-141$.

Barrow J. 1836. Sketch of the surveying voyages of this Majesty's Ship Adventure and Beagle, 1825-1836: commanded by Captain P.P. King, P. Stokes, and R. FitzRoy, Royal Navy. Journal of Geological Society of London 6: 311-343.

Broitman B, S Navarrete, F Smith \& S Gaines. 2001. Geographic variation of Southeastern Pacific intertidal communities. Marine Ecology Progress Series 224: 21-34.

Camus P \& NA Lagos. 1996. Variabilidad espacio-temporal del reclutamiento de ensambles sésiles del intermareal rocoso del norte de Chile. Revista Chilena de Historia Natural 69: 193- 204.

Castilla JC. 1981. Perspectivas de investigación en estructura y dinámica de comunidades intermareales rocosas de Chile central. II. Depredadores de alto nivel trófico. Medio Ambiente 5: 190-215.

Castilla JC \& D Oliva. 1990. Ecological consequences of coseismic uplift on the intertidal kelp belts of Lessonia nigrescens in central Chile. Estuarine Coastal and Shelf Science 31: 45-56.

Castilla JC, PH Manríquez \& A Camaño. 2010. Effects of rocky shore coseismic uplift and the 2010 Chilean megaearthquake on intertidal biomarker species. Marine Ecology Progress Series 418: 17-23.

Cerda M \& JC Castilla. 2001. Diversidad y biomasa de macroinvertebrados en matrices intermareales del tunicado Pyura praeputialis (Heller, 1878) en la Bahía de Antofagasta, Chile. Revista Chilena de Historia Natural 74: 841-853. 
Cisternas M, BF Atwater, F Torrejon, Y Sawai, G Machuca, M Lagos, A Eipert, C Youlton, I Salgado, T Kamataki, M Shishikura, CP Rajendran, JK Malik, Y Rizal \& M Husni. 2005. Predecessors of the giant 1960 Chile earthquake. Nature 437: 404-407.

Darwin C. 1851. Geological observations on coral reefs, volcanic islands and on South, America, 768 pp. Smith, Elder and Company, London.

Farías M, G Vargas, A Tassara, S Carretier, S Baize, D Melnick \& K Bataille. 2010. Land-level changes produced by the Mw 8.82010 Chile earthquake. Science 329: 916.

Jaramillo E, JE Dugan, DM Hubbard, D Melnick, M Manzano, C Duarte, C Campos \& R Sanchez. 2012. Ecological implications of extreme events: Footprints of the 2010 earthquake along the Chilean coast. PLoS ONE 7(5): e35348. <doi:10.1371/journal.pone.0035348>

Lagos NA, FJ Tapia, SA Navarrete \& JC Castilla. 2007. Spatial synchrony in the recruitment of intertidal invertebrates along the coast of central Chile. Marine Ecology Progress Series 350: 29-39.

Lee RV. 2010. Darwin’s Earthquake. Revista Médica de Chile 138: 897-901.

Lomnitz C. 2004. Major earthquakes of Chile: A historical survey 1535-1960. Seismological Research Letters 75: 368378.

Madariaga R, M Marianne, C Vigny \& J Campos. 2010. Central Chile finally breaks. Science 328: 181-182.

Meneses I. 1993. Vertical distribution of coralline algae in the rocky intertidal of northern Chile. Hydrobiologia 260/261: 121-129.

Melnick D, M Cisternas, M Moreno \& R Norambuena. 2012. Estimating coseismic coastal uplift with an intertidal mussel: calibration for the 2010 Maule Chile earthquake (Mw= 8.8). Quaternary Science Reviews 42: 29-42.

Montecinos A, B Britman, S Faugeron, P Hye, F Tellier \& M Guillemin. 2012. Species replacement along a linear coastal habitat: phylogeography and speciation in the red alga Mazzaella laminarioides along the South East Pacific. BioMedCentral, Evolutionary Biology 12: 97.

Morton RA, G Gelfenbaum, ML Buckley \& BM Richmond. 2011. Geological effects and implications of the 2010 tsunami along the central coast of Chile. Sedimentary Geology 242: 34-51.

Ortega KJ, CA Sáez \& EC Macaya. 2014. Changes in invertebrate assemblages inhabiting Lessonia spicata
(Phaeophyceae) holdfasts after the 2010 earthquakemediated coastal uplift in Chile. Revista de Biología Marina y Oceanografía 49: 129-134.

Ortlieb L, S Barrientos \& N Guzmán. 1996. Coseismic coastal uplift and coralline algae record in northern Chile: the 1995 Antofagasta earthquake case. Quaternary Science Review 15: 949-960.

Plafker G \& JC Savage. 1970. Mechanism of the Chilean earthquake of May 21 and 22, 1960. Bulletin Geological Society of America 81: 1001-1030.

Quezada J, E Jaque, A Belmonte, A Fernández, D Vásquez \& C Martínez. 2010. Movimientos cosísmicos verticales y cambios geomorfológicos generados durante el terremoto Mw= 8,8 del 27 de Febrero de 2010 en el centro-sur de Chile. Revista Geográfica del Sur 2: 11-45.

Ramírez-Herrera MT \& JJZ Orozco. 2002. Coastal uplift and mortality of coralline algae caused by a $6.3 \mathrm{Mw}$ earthquake, Oaxaca, México. Journal of Coastal Research 18: 75-81.

Rodil IF, E Jaramillo, DM Hubbard, JE Dugan, D Melnick \& C Velasquez. 2015. Responses of dune plant communities to continental uplift from a major earthquake: sudden releases from coastal squeeze. PLoS One 10(5), e0124334. <https://doi.org/10.1371/journal.pone.0124334>

Rodil IF, E Jaramillo, E Acuña, M Manzano \& C Velasquez. 2016. Long-term responses of sandy beach crustaceans to the effects of coastal armouring after the 2010 Maule earthquake in South Central Chile. Journal of Sea Research 108: 10-18.

Ruegg JC, A Rudloff, C Vigny, R Madariaga, JB de Chabalier, J Campos, E Kausel, S Barrientos \& D Dimitrov. 2009. Interseismic strain accumulation measured by GPS in the seismic gap between Constitución and Concepción in Chile. Physics of the Earth and Planetary Interiors 175: 78-85.

Santelices B. 1991. Littoral and sublittoral communities of continental Chile. In: Mathieson AC \& PH Nienhius (eds). Intertidal and littoral ecosystem. Ecosystem of the World 24: 347-369. Elsevier, Amsterdam.

Vargas G, M Farias, S Carretier, A Tassara, S Baize \& D Melnick. 2011. Coastal uplift and tsunami effects associated to the $2010 \mathrm{Mw}$ 8.8 Maule earthquake in Central Chile. Andean Geology 38: 219-238.

Wong PP. 2009. Impacts and recovery from a large tsunami: coast of Aceh. Polish Journal of Environmental Studies 18: 5-16.

Recibido el 29 de septiembre de 2017 y aceptado el 22 de marzo de 2018 Editor: Claudia Bustos D. 
Tabla S1. Localización de los sitios de estudio en la costa de las regiones del Maule, Biobío, Los Ríos y Los Lagos, Chile / Location of the study sites along the coastal zones of the regions of Maule, Biobío, Los Ríos and Los Lagos, Chile

\begin{tabular}{|c|c|c|c|}
\hline Región y áreas & Sitios & $\begin{array}{l}\text { Latitud } \\
\text { (S) }\end{array}$ & $\begin{array}{l}\text { Longitud } \\
\text { (W) }\end{array}$ \\
\hline \multirow[t]{3}{*}{ MAULE } & Boyeruca & $34^{\circ} 41^{\prime} 22,2^{\prime \prime}$ & $72^{\circ} 03^{\prime} 39,1^{\prime \prime}$ \\
\hline & Lipimávida & $34^{\circ} 49^{\prime} 49,1^{\prime \prime}$ & $2^{\circ} 08^{\prime} 42.3^{\prime \prime}$ \\
\hline & Pelluhue & $35^{\circ} 48^{\prime} 56,1^{\prime \prime}$ & $72^{\circ} 35^{\prime} 10,7^{\prime \prime}$ \\
\hline BIOBÏO - al norte de la & Cocholhue & $36^{\circ} 35^{\prime} 53,3^{\prime \prime}$ & $72^{\circ} 58^{\prime} 31,9^{\prime \prime}$ \\
\hline \multirow[t]{3}{*}{ Península de Arauco } & Lenga & $36^{\circ} 45^{\prime} 52,0^{\prime \prime}$ & $73^{\circ} 10^{\prime} 29,7^{\prime \prime}$ \\
\hline & Chome & $36^{\circ} 46^{\prime} 07,0^{\prime \prime}$ & $73^{\circ} 12^{\prime} 29,9^{\prime \prime}$ \\
\hline & Maule & $37^{\circ} 00^{\prime} 36.2^{\prime \prime}$ & $73^{\circ} 10^{\prime} 59.1 "$ \\
\hline BIOBÍO - Península de & Colcura & $37^{\circ} 07^{\prime} 06,7^{\prime}$, & $73^{\circ} 0,1^{\prime} 17,8^{\prime \prime}$ \\
\hline \multirow[t]{6}{*}{ Arauco } & Lavapié & $37^{\circ} 09^{\prime} 02,4^{\prime \prime}$ & $73^{\circ} 34^{\prime} 24,4^{\prime \prime}$ \\
\hline & Llico & $37^{\circ} 11^{\prime} 03 \prime \prime$ & $73^{\circ} 33^{\prime} 50,6^{\prime \prime}$, \\
\hline & Lebu Norte & $37^{\circ} 34^{\prime} 48,2^{\prime \prime}$ & $73^{\circ} 38^{\prime} 34,3^{\prime \prime}$ \\
\hline & Lebu Sur & $37^{\circ} 36^{\prime} 38^{\prime \prime}$ & $73^{\circ} 39^{\prime} 50^{\prime \prime}$ \\
\hline & Quidico & $38^{\circ} 14^{\prime} 32,5^{\prime \prime}$ & $73^{\circ} 29^{\prime} 32^{\prime \prime}$ \\
\hline & Tirúa & $38^{\circ} 20^{\prime} 28,3^{\prime \prime}$ & $73^{\circ} 30^{\prime} 27,7^{\prime \prime}$ \\
\hline \multirow[t]{7}{*}{ BIOBÍO - Isla Santa María } & Punta Norte & $36^{\circ} 58^{\prime} 06,1^{\prime \prime}$ & $73^{\circ} 31^{\prime} 25^{\prime \prime}$ \\
\hline & Gaviotas & $36^{\circ} 58^{\prime} 37,6^{\prime \prime}$ & $73^{\circ} 32^{\prime} 03,9^{\prime \prime}$ \\
\hline & Caleta Hernández & $36^{\circ} 58^{\prime} 29,8^{\prime \prime}$ & $73^{\circ} 31 ' 20,9$ '” \\
\hline & Tres Cuevas & $36^{\circ} 59^{\prime} 10,2^{\prime \prime}$ & $73^{\circ} 31^{\prime} 06,2^{\prime \prime}$ \\
\hline & Dolores & $37^{\circ} 01 ' 20,4^{\prime \prime}$ & $73^{\circ} 33^{\prime} 08,9^{\prime \prime}$ \\
\hline & Las Torres & $37^{\circ} 04^{\prime} 09,4^{\prime \prime}$ & $73^{\circ} 31^{\prime} 23,5^{\prime \prime}$ \\
\hline & La Celina & $37^{\circ} 04^{\prime} 03,6^{\prime \prime}$ & $73^{\circ} 30^{\prime} 52,1^{\prime \prime}$ \\
\hline \multirow[t]{6}{*}{ BIOBÍO - Isla Mocha } & Arveja & $38^{\circ} 19^{\prime} 3,6^{\prime \prime}$ & $73^{\circ} 57^{\prime} 26,3^{\prime \prime}$ \\
\hline & Muelle & $38^{\circ} 20^{\prime} 8,1^{\prime \prime}$ & $73^{\circ} 54 ' 24,6^{\prime \prime}$ \\
\hline & El Natre & $38^{\circ} 21^{\prime} 14,0^{\prime \prime}$ & $73^{\circ} 56^{\prime} 38,3^{\prime \prime}$ \\
\hline & El Faro & $38^{\circ} 22^{\prime} 10,5^{\prime \prime}$ & $73^{\circ} 56^{\prime} 49,3^{\prime \prime}$ \\
\hline & El Santiago & $38^{\circ} 23$ ' $53,8^{\prime \prime}$ & $73^{\circ} 52^{\prime} 23,1^{\prime \prime}$ \\
\hline & El Saco & $38^{\circ} 24^{\prime} 59,3 \prime \prime$ & $73^{\circ} 53^{\prime} 49,2^{\prime \prime}$ \\
\hline \multirow[t]{2}{*}{ LOS RÍOS - Valdivia } & Centinilla & $39^{\circ} 48^{\prime} 16,4^{\prime \prime}$ & $73^{\circ} 23^{\prime} 58,4^{\prime \prime}$ \\
\hline & Loncoyén & $39^{\circ} 49^{\prime} 01,4^{\prime \prime}$ & $73^{\circ} 24^{\prime} 07,1^{\prime \prime}$ \\
\hline \multirow[t]{3}{*}{ LOS LAGOS - Osorno } & Pucatrihue & $40^{\circ} 32^{\prime} 37,5^{\prime \prime}$ & $73^{\circ} 43^{\prime} 01,5^{\prime \prime}$ \\
\hline & Maicolpué & $40^{\circ} 35^{\prime} 11,7^{\prime \prime}$ & $73^{\circ} 44^{\prime} 13,1^{\prime \prime}$ \\
\hline & Tril Tril & $40^{\circ} 36^{\prime} 53,6^{\prime \prime}$ & $73^{\circ} 45^{\prime} 12,3^{\prime \prime}$ \\
\hline
\end{tabular}

Tabla S2. Abreviaturas utilizadas en el texto de este estudio / Abbreviations used in the text of this study

\begin{tabular}{lll}
\hline $\begin{array}{l}\text { 27F: Fecha del terremoto y tsunami } \\
27 \text { de febrero del } 2010\end{array}$ & LEBN: Lebu Norte & MU: Muelle (IM) \\
$\begin{array}{l}\text { Ls: Limite superior } \\
\text { BO: Boyeruca }\end{array}$ & LEBS: Lebu Sur & ELN: El Natre (IM) \\
LI: Lipimávida & QUID: Quidico & ELS: El Santiago (IM) \\
PE: Pelluhue & TIR: Tirúa & ES: El Saco Este (IM) \\
COCHO: Cocholhue & PNO: Punta Norte (ISM) & FA: Faro (IM) \\
LE: Lenga & GAV: Gaviota (ISM) & CEN: Centinilla \\
CHOME: Chome & CHERN: Caleta Hernández (ISM) & LON: Loncoyén \\
MA: Maule & TCUE: Tres Cuevas (ISM) & PUC: Pucatrihue \\
CO: Colcura & DOL: Dolores (ISM) & MAICO: Maicolpué \\
LAV: Lavapié & LTORR: Las Torres (ISM) & TRIL: Tril Tril \\
LLI: Llico & LCEL: La Celina (ISM) & \\
\hline
\end{tabular}

